\title{
A NEW WATERMARKING SCHME AGAINST LOCAL ATTACKS
}

\author{
Neethu Raveendran.K ${ }^{1}$ \\ ${ }^{I}$ PG Student, Department of Computer Science and Engineering Specialization in Information System, Federal Institute of \\ Science and Technology, Kerala, India
}

\begin{abstract}
Many watermarking schemes are robust against common image processing attacks like Rotation Scaling Translation (RST) attack. In this paper, presents a blind watermarking scheme against local attacks. First, proposes a new globally and locally feature transform called Local Daisy feature transform (LDFT). Then, partition this invariant geometric space using Binary Space Partition Tree (BSP). The location of every single pixel is in variable to geometrical distortions like rotation, scaling, translation and resistant against cropping. Lastly, Logarithmic quantization index modulation (LQIM) watermarking embedding method is used to embed watermarking sequence bit by bit into leaf nodes of binary space partition tree. This method overcomes the drawbacks of feature based watermarking method. The watermark Extraction is similar to Watermark Embedding. In Extraction process also LDFT space is created and partitioned using BSP tree.
\end{abstract}

Keywords: Binary Space Partition tree, Local Daisy feature transform, Logarithmic quantization index modulation

\section{INTRODUCTION}

Digital watermarking is a process of embedding information into digital multimedia, so that information can be detected for various purposes. In the past ten years, attacks against image watermarking system have become increasingly complicated with the development of watermarking techniques. Every geometric attack is defined by a set of parameters that determines the operation performed over the target image (for example, a rotation angle applied to the sampling grid characterizes a rotation attack). An affine transformation preserves lines and parallelism. In cropping, the attacker extracts a certain region of interest of the image while discarding the other portions. Obviously, we can't invert cropping because it incurs a permanent loss of information about the discarded pixels. Attacks can be classified into two categories: global and local. A global transformation is an operation uniquely defined by a set of operational parameters and performed over an image's entire set of pixels, that is, the same operation, under the same parameters, affects all of the image pixels. On the other hand, localized transformations affect subsets of the image, allowing an attacker to apply distinct operations under different parameters over each image subset.

Global attacks include Rotation, Scaling and Translation (RST) attack, Linear Geometric Transform, shearing and other affine transforms. The parameters of global attack are not complicated. There are many countermeasures are available for global attacks. M. Barni proposed an exhaustive search for the watermark in the space including a set of acceptable attack parameters [1]. Ruanaidh and Pun proposed Fourier-Mellin transform which is invariant to global RST transformation [2]. Image normalization algorithm achieves its robustness by both embedding and detecting the watermark message in the normalized image [3]. Another countermeasure is a blind watermarking scheme using geometrically invariant image features. The detection of the watermark can be conducted with the help of image features [4]. Local attacks include Random Bending Attack, C-LPCD (Constrained Local Permutation with Cancelation and Duplication) etc. Very few schemes are available against local attacks.

In this paper, presents a blind watermarking scheme against local attacks. A new globally and locally feature transform called Local Daisy feature transform. Then, partition this invariant geometric space using Binary Space Partition Tree. The location of every single pixel is in variable to geometrical distortions like rotation, scaling, translation and resistant against cropping. Lastly, Logarithmic quantization index modulation watermarking embedding method is used to embed watermarking sequence bit by bit into leaf nodes of binary space partition tree.

\section{SYSTEM ARCHITECTURE}

The watermark embedding process consists of three main steps: 1) constructing the feature space with Local Daisy Feature Transform; 2) partitioning the LDFT space with the Binary Space Partition tree; and 3) embedding watermark information by Logarithmic Quantization Index Modulation watermark embedding method. The watermark extracting process resembles watermark embedding, which comprises three main steps: 1) constructing the feature space of LDFT; 2) partitioning the LDFT space with the BSP tree; and 3) watermark extraction. The proposed watermarking scheme is a blind watermarking scheme. 


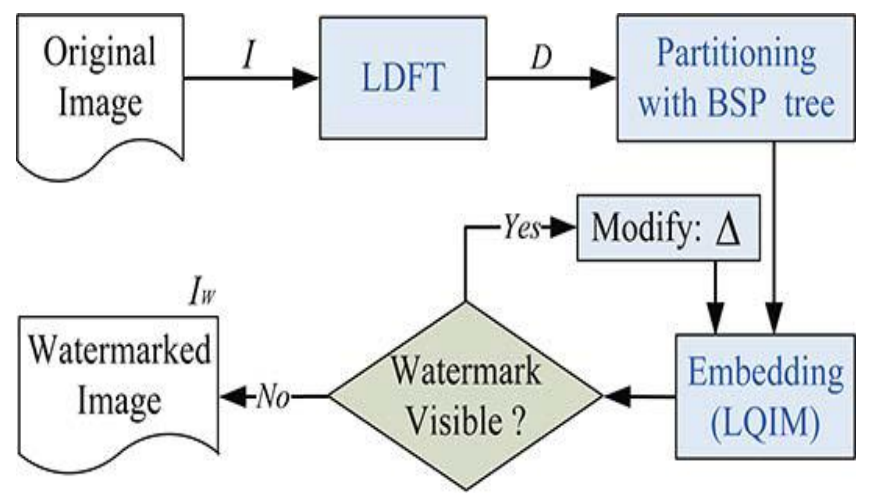

Fig -1: System Architecture

\section{PHASES}

There are four phases which are listed below

- $\quad$ Proposed LDFT

- $\quad$ Partitioning LDFT space using BSP tree

- Watermark embedding

- Watermark Extraction

\subsection{Proposed LDFT}

Most Of the watermarking schemes are robust against global attacks. A new invariant feature transform called Local daisy Feature Transform is proposed in this section. LDFT is based in daisy descriptor [5]. Daisy descriptor is not RST in variant. But this LDFT is not only globally but also locally invariant. In order to create LDFT there are several steps. Initially we need to compute the characteristic scale map of the image. LoG operator is used to obtain the characteristics scale map of the image. This scale map varies with respect to image scaling. Then compute orientation map in every quantized direction this orientation map is then convolved with Gaussian Kernels of different standard deviation. After this convolution we get convolved orientation maps. Then normalize the convolved orientation maps. Now compute the Euclidean distance between normalized vector and the original vector. Finally assign a consistent orientation to each pixel based on image properties. Orientation assignment is done using Histogram of oriented gradients (HOG) [6].

\subsection{Partitioning LDFT Space using BSP Tree}

In this section we are partitioning the geometrically invariant LDFT space using BSP tree [7]. BSP tree construction is a process which takes a subspace and partitions it by any hyper plane that intersects the interior of that subspace. It generates two new subspaces that can be further partitioned by recursive processes. Initially we select a partition plane to divide the LDFT space. To improve the security we can randomize the order of the selection of the partition plane using a secret key. In order to achieve a balanced BSP tree, set a threshold value. The threshold value is computed as the average values of all the partition planes of the image. Then partition the space using the plane. If the subset is under the partition plane and the value of the feature vector is less than threshold value, the subset is the left child of tree, else right child of tree. This process continues until the no of leaf nodes is equal to the length of the watermark sequence.

\subsection{Watermark Embedding}

Watermarking Sequence is embedded bit by bit into leaf node from left to right using Logarithmic Quantization Index Modulation (LIQM) [8]. Initially, using a compression function the host signal is transformed into logarithmic domain. The watermark data is embedded using uniform quantization. Then using the inverse function, the quantized signal is transformed into the original domain

\subsection{Watermark Extraction}

Extraction is similar to embedding. Initially create the LDFT space and partition it using BSP tree. Now determine the embedded watermark bit using Euclidean distance decoder.

\section{CONCLUSIONS}

A new watermarking scheme against local attack is proposed. Initially a new feature transform called Local Daisy feature transforms is proposed. LDFT is inspired by daisy descriptor. LDFT is invariant to geometrical attacks. Then LDFT space is partitioned using BSP trees. Watermark bits are embedded using Logarithmic quantization index modulation method.

\section{REFERENCES}

[1]. M. Barni, "Effectiveness of exhaustive search and template matching against watermark desynchronization", IEEE Signal Process, vol 12 no.2, Feb 2005

[2]. J. Ruanaidh and T. Pun, "Rotation, scale and translation invariant spread spectrum digital image watermarking", signal process, vol.66, no.3, May 1998

[3]. P. Dong, J.G Brankov, N.P. Galatsanos, Y.Yang, and F. Davoine, "digital watermarking robust to geometric distortion" IEEE Trans, Image Process,. Vol.11 no.9, Sep 2002

[4]. P. Bas, J. Chassey and B. Macq, "Geometrically invariant watermarking using feature points", IEEE Trans, Image Process, vol.51, no., apr 2003.

[5]. E. Tola, V. Lepetit and P.Fua,'Daisy: An efficient dense descriptor applied to wide-baseline stereo", IEEE Trans. Pattern Anal. Mach. Intell, vol 32,no5,may 2010

[6]. N.Dalal and B. Triggs, "Histogramof oriented gradients for human detection" in Proc IEEE Comput. Soc. Conf. Comput. Vis In Pattern Recognition, June 2005

[7]. M.Abrash,"BSP Trees",Dr.Dobbs sourcebook, vol 20, may 1995

[8]. N.K. Kalantari and S.M Ahadi, "a logarithmic quantization index modulation for perceptually better data hiding”, IEEE Trans. Image Processing, vol 19, june 2010 